\title{
Early Heparin Administration Reduces Risk for Left Atrial Thrombus Formation during Atrial Fibrillation Ablation Procedures
}

\author{
Stefan Asbach, Jürgen Biermann, Christoph Bode, and Thomas S. Faber \\ Department of Cardiology and Angiology, University Hospital Freiburg, Hugstetter Straße 55, 79106 Freiburg, Germany \\ Correspondence should be addressed to Stefan Asbach, stefan.asbach@uniklinik-freiburg.de
}

Received 16 February 2011; Revised 20 April 2011; Accepted 5 May 2011

Academic Editor: J. Brugada

Copyright (C) 2011 Stefan Asbach et al. This is an open access article distributed under the Creative Commons Attribution License, which permits unrestricted use, distribution, and reproduction in any medium, provided the original work is properly cited.

\begin{abstract}
Objective. Despite the use of anticoagulation during left atrial (LA) ablation procedures, ischemic cerebrovascular accidents (CVAs) are recognized as a serious complication. Heparin is usually given after safe transseptal access has been obtained, resulting in a short unprotected dwell time of catheters within the LA, which may account for CVAs. We investigated the frequency of CVAs and LA thrombus formation as detected by intracardiac ultrasound (ICE) depending on the timing of heparin administration. Methods and Results. Sixty LA ablation procedures with the use of ICE were performed in 55 patients. Patients were grouped by heparin administration after (Group I, $n=13$ ) and before (Group II, $n=47$ ) transseptal access. Group I patients were younger (56.6 \pm 13.7 versus $65.9 \pm 9.9$ years, $P=.01)$; other clinical and echocardiographic characteristics did not differ between groups. Early thrombus formation was observed in $2(15.4 \%)$ of group I patients as compared to $0 \%$ of group II patients $(P=.04)$. One CVA $(2.1 \%)$ occurred in one group II patient without prior thrombus detection, and none occurred in group I patients $(P=\mathrm{ns})$. Conclusion. Early administration of heparin reduces the risk of early intracardiac thrombus formation during LA ablation procedures. This did not result in reduced rate of CVAs.
\end{abstract}

\section{Introduction}

After the initial description of the role of the pulmonary veins in initiating and maintaining atrial fibrillation $(\mathrm{AF})$ [1], increasing clinical evidence of efficacy and safety of left atrial ablation has been gathered [2]. Consequently, catheter ablation of $\mathrm{AF}$ is performed in increasing numbers and centers $[3,4]$. Despite this increase in experience and the rapid development of novel tools to facilitate the procedure and improve success rates, the procedure remains complex mainly due to (a) the need for transseptal access, (b) the variable left atrial anatomy, and (c) the possible risk of serious periprocedural complications, including cerebral embolism.

A major concern is the development and embolism of intracardiac thrombi during the procedure with the possible risk of stroke. Therefore, the use of heparin with an activated clotting time (ACT) of 300-400 s is mandatory and endorsed by current guidelines [5]. Despite this, the reported rate of peri-interventional cerebrovascular accidents ranges from 0 to $7 \%$ [5]. The administration of heparin is commonly started after safe transseptal access has been obtained in order not to aggravate a possible, transseptal, puncture-related pericardial effusion. This however results in a short dwell time of foreign material within the left atrium without effective anticoagulation, which may be sufficient for thrombus formation and which thus may account for the still evident risk of embolism. Therefore, we sought to evaluate the possible benefits of early versus late heparin administration in regard to intracardiac thrombus formation as assessed by intracardiac echocardiography (ICE). Intracardiac echocardiography can be safely used throughout invasive procedures to visualize intracardiac structures, monitor transseptal puncture and intracardiac catheters as well as energy delivery, early detect complications, and possibly avoid adverse events [6-8]. The value of ICE in monitoring the left atrium for intracardiac thrombi has previously been demonstrated and is therefore used in this study to investigate the presence of thrombi depending on peri-interventional anticoagulant treatment. 


\section{Materials and Methods}

2.1. Patients. In this observational study, we included 60 consecutive AF ablation procedures in 55 patients (5 Re-Do procedures), which were performed with the use of ICE. All patients suffered from symptomatic, drug-refractory AF. Please refer to Table 1 for patient characteristics. One day prior to the ablation procedure, transesophageal echocardiography (TEE) was performed in all patients to rule out intracardiac thrombi. Emptying velocity of the left atrial appendage was determined by pulsed wave Doppler during TEE. All patients underwent pulmonary vein isolation without additional lesion sets after written informed consent was obtained.

2.2. Procedure. Oral anticoagulation was stopped prior to the ablation procedure until the international normalized ratio dropped below 1.5. Overlapping low molecular weight heparin was given in body-weight adjusted doses. All procedures were performed under conscious sedation. Vascular access was obtained via the left femoral vein for insertion of a decapolar, $4 \mathrm{~F}$ coronary sinus catheter (Inquiry, St. Jude Medical, USA), and the 10F ICE catheter (AcuNav, Siemens, Germany). The right atrium, interatrial septum, left atrium, mitral and aortic valves, left atrial appendage, and the left and right pulmonary veins were visualized by ICE. Subsequently, transseptal sheaths were advanced via the right femoral vein into the right atrium, and, after transseptal access, into the left atrium. Transseptal sheaths were continuously flushed with heparinised saline. Catheters for mapping and ablation were advanced via the transseptal sheaths into the left atrium. We used either a CARTO guided approach $(n=26)$ with a circular mapping catheter (Lasso, Biosense Webster, Diamond Bar, USA) and an open irrigated tip ablation catheter (NaviStar ThermoCool, Biosense Webster, Diamond Bar, USA), or a duty-cycled circular mapping and ablation catheter $(n=34$, Pulmonary Vein Ablation Catheter, Ablation Frontiers, USA). Heparin was administered after transseptal access as a bolus of $100 \mathrm{U} / \mathrm{kg}$ in the first 13 procedures (Group I, "late heparin") and in all following 47 procedures (Group II, "early heparin") as a bolus of 4000-5000 U after puncture of the femoral veins but before transseptal access, followed by another bolus after transseptal access with a cumulative target dose of $100 \mathrm{U} / \mathrm{kg}$. Heparin was then continuously administered to achieve an ACT of 300-400 s. ACT was measured $15 \mathrm{~min}$ after transseptal access and every 15-30 min thereafter. Catheters were guided by an electroanatomical map whenever available, by intermittent fluoroscopy and by continuous monitoring of the ICE images. Special attention was paid to verify correct and stable catheter positions in the ICE images and to continuously monitor for possible side effects such as pericardial effusion, intracardiac thrombi, and showers of bubbles suggestive for tissue overheating. Endpoint of the procedure was pulmonary vein isolation as determined by loss of pulmonary vein potentials and entrance block.

2.3. Statistics. All variables are presented \pm standard deviation. Data were analysed for Gaussian distribution using the
D'Agostino-Pearson normality test. Subsequent comparison was performed using the Mann-Whitney test for variables with non-Gaussian distribution or the unpaired $t$-test for gaussian distribution, respectively. Fishers test was performed for comparison of discrete variables. A $P$-value $<.05$ was considered significant.

\section{Results and Discussion}

3.1. Patient Characteristics. Patient characteristics are shown in Table 1. Patients in group I were younger (56.6 \pm 13.7 versus $65.9 \pm 9.9$ years, $P=.01)$. Other baseline factors such as gender $(76.9 \%$ versus $61.7 \%$ males, $P=.51)$, type of $\mathrm{AF}$ (46.2\% versus $65.9 \%$ paroxysmal, $P=.21$ ), $\mathrm{CHADS}_{2}$-Score $(1.5 \pm 1.1$ versus $1.5 \pm 1.1, P=.91)$, history of cerebrovascular event $(30.8 \%$ versus $12.8 \%, P=.20)$, hypertension $(76.9 \%$ versus $70.2 \%, P=.74)$, diabetes $(0 \%$ versus $12.8 \%$, $P=.32)$, and coronary artery disease (15.4\% versus $27.7 \%$, $P=.48)$ did not differ between groups. Echocardiographic parameters such as left atrial diameter $(46.9 \pm 9.0$ versus $45.1 \pm 7.0 \mathrm{~mm}, P=.65)$, left ventricular ejection fraction $(51.2 \pm 9.8$ versus $51.7 \pm 8.8 \%, P=.74)$, left ventricular enddiastolic diameter $(51.4 \pm 5.6$ versus $52.7 \pm 9.4 \mathrm{~mm}, P=.98)$, wall thickness of septum $(10.8 \pm 1.2$ versus $10.7 \pm 1.5 \mathrm{~mm}$, $P=.61)$, posterior wall $(10.5 \pm 1.9$ versus $10.8 \pm 1.3 \mathrm{~mm}$, $P=.62)$, and maximal emptying velocity of the left atrial appendage $(41.2 \pm 11.6$ versus $48.5 \pm 17.1 \mathrm{~cm} / \mathrm{s})$ also did not differ between groups.

3.2. Observations by Intracardiac Ultrasound. In $2 / 13$ (15.4\%) group I patients as compared to $0 \%$ of group II patients, intracardiac thrombi were detected $(P=.04)$. Classification of thrombus was made because of independent mobility and tissue characteristics consistent with thrombus. One thrombus adhered to a circular mapping catheter (Figure 1), the other thrombus was visualized adherent to a transseptal sheath (Figure 2). In both cases, thrombi were detected early after transseptal access and before radiofrequency energy delivery. Disruption of the endocardial surface by radiofrequency ablation thus cannot be responsible for thrombus formation. The thrombus attached to the transseptal sheath was detected before administration of heparin, whereas the thrombus which was attached to the circular mapping catheter was detected after heparin administration. In the latter case, ACT measured immediately after thrombus detection was $289 \mathrm{~s}$. These two patients did not differ regarding baseline parameters from the whole group. In both cases, additional heparin was given to achieve an ACT > $300 \mathrm{~s}$. Sheaths and catheters were retracted to the right atrium while strong suction was applied to the sheath's sidearm, carefully flushed, and another transseptal puncture performed. No thrombi could be detected in the aspirate, neither were any clinical signs suggestive of central, peripheral, or pulmonary embolism noted.

Based on the frequent observation of thrombi, we changed the anticoagulatory regimen to administration of heparin before transseptal access and a subsequent bolus after transseptal access to reach a target dose of $100 \mathrm{U} / \mathrm{kg}$. In all following procedures, no intracardiac thrombi could 
TABLe 1: Patient characteristics.

\begin{tabular}{|c|c|c|c|c|}
\hline & All & $\begin{array}{c}\text { Group I } \\
\text { "late heparin" }\end{array}$ & $\begin{array}{c}\text { Group II } \\
\text { "early heparin" }\end{array}$ & $P$ \\
\hline$n$ & $60(100 \%)$ & $13(21.7 \%)$ & $47(78.3 \%)$ & \\
\hline Age (years) & $63.9 \pm 11.3$ & $56.6 \pm 13.7$ & $65.9 \pm 9.9$ & .01 \\
\hline Male gender $(n)$ & $39(65.0 \%)$ & $10(76.9 \%)$ & $29(61.7 \%)$ & .51 \\
\hline Paroxysmal AF $(n)$ & $37(61.7 \%)$ & $6(46.2 \%)$ & $31(65.9 \%)$ & .21 \\
\hline $\mathrm{CHADS}_{2}$ Score & $1.5 \pm 1.1$ & $1.5 \pm 1.1$ & $1.5 \pm 1.1$ & .92 \\
\hline $\mathrm{Hx} \operatorname{CVA}(n)$ & $10(16.7 \%)$ & $4(30.8 \%)$ & $6(12.8 \%)$ & .2 \\
\hline Hypertension $(n)$ & $43(71.7 \%)$ & $10(76.9 \%)$ & $33(70.2 \%)$ & .74 \\
\hline Diabetes $(n)$ & $6(10 \%)$ & 0 & $6(12.8 \%)$ & .32 \\
\hline $\mathrm{CAD}$ & $15(25 \%)$ & $2(15.4 \%)$ & $13(27.7 \%)$ & .48 \\
\hline \multicolumn{5}{|l|}{ Echo-Parameters } \\
\hline LV-EF (\%) & $51.6 \pm 8.9$ & $51.2 \pm 9.8$ & $51.7 \pm 8.8$ & .74 \\
\hline LA diameter $(\mathrm{mm})$ & $45.5 \pm 7.5$ & $46.9 \pm 9.0$ & $45.1 \pm 7.0$ & .65 \\
\hline $\operatorname{LVEDD}(\mathrm{mm})$ & $52.4 \pm 8.7$ & $51.4 \pm 5.6$ & $52.7 \pm 9.4$ & .98 \\
\hline IVSd (mm) & $10.7 \pm 1.4$ & $10.8 \pm 1.2$ & $10.7 \pm 1.5$ & .61 \\
\hline PWd (mm) & $10.7 \pm 1.5$ & $10.5 \pm 1.9$ & $10.8 \pm 1.3$ & .62 \\
\hline LAA Vmax $(\mathrm{cm} / \mathrm{s})$ & $47.0 \pm 16.3$ & $41.2 \pm 11.6$ & $48.5 \pm 17.1$ & .24 \\
\hline Initial ACT (s) & $290.1 \pm 67.8$ & $244.3 \pm 40.7$ & $301.7 \pm 68.7$ & .002 \\
\hline Mean ACT (s) & $315.0 \pm 43.7$ & $297.9 \pm 40.9$ & $319.4 \pm 43.7$ & .11 \\
\hline
\end{tabular}

AF: atrial fibrillation, Hx CVA: history of cerebrovascular event, CAD: coronary artery disease, LV-EF: left ventricular ejection fraction, LA: left atrium, LVEDD: left ventricular end-diastolic diameter, IVSd: interventricular septum thickness, PWd: posterior wall thickness, LAA Vmax: left atrial appendage maximal flow velocity, and ACT: activated clotting time.

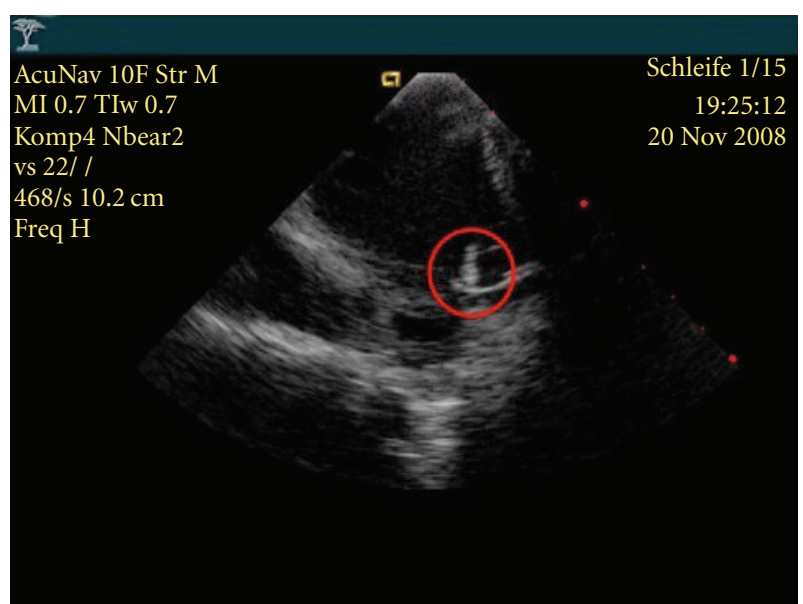

FIGURE 1: The circular mapping catheter can clearly be visualized in the antrum of the left superior pulmonary vein. Attached to the inferior part of the ring of electrodes, a mobile thrombus can clearly be seen (encircled). Immediately measured ACT was $289 \mathrm{~s}$.

be observed. Due to the fact that the first ACT was measured $15 \mathrm{~min}$ after transseptal access rather than heparin administration, it was significantly shorter in group I than in group II $(244.3 \pm 40.7$ versus $301.7 \pm 68.7 \mathrm{~s}, P=.002)$. Mean ACTs during the procedure did not differ between groups $(297.9 \pm 40.9$ versus $319.4 \pm 43.7, P=.11)$.

3.3. Complications. We observed one cardiac tamponade with the need for pericardiocentesis, which occurred during

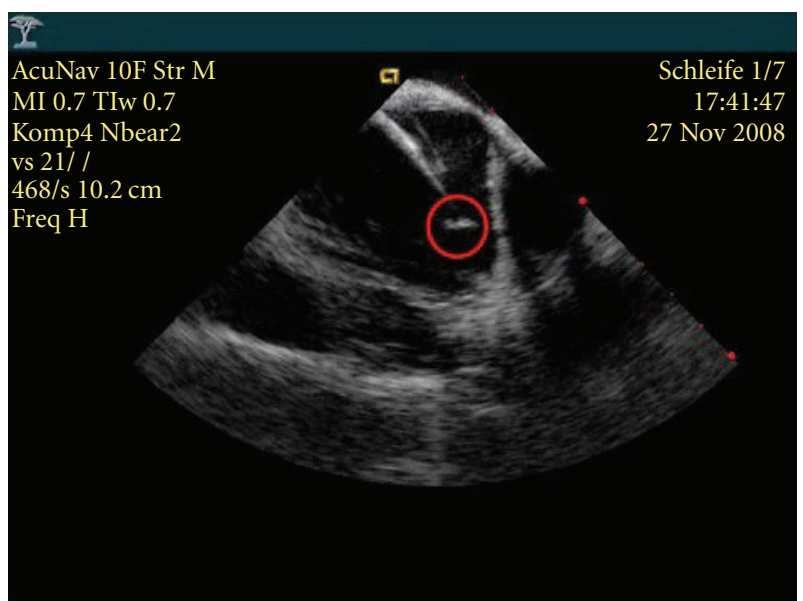

FIgURE 2: A mobile thrombus (encircled) is attached to the tip of the transseptal sheath before the advancement of a catheter and before heparin administration.

ablation on the ridge between the left atrial appendage and the left superior pulmonary vein. The patient recovered without sequelae. No tamponade occurred during transseptal puncture.

One periprocedural stroke occurred in one group II patient $(1 / 47 ; 2.1 \%)$ in whom we did not observe an intracardiac thrombus formation during ICE monitoring. The patient complained about difficulties speaking without any further neurologic deficits. While initial examination by the neurologist and cerebral imaging by computed tomography 


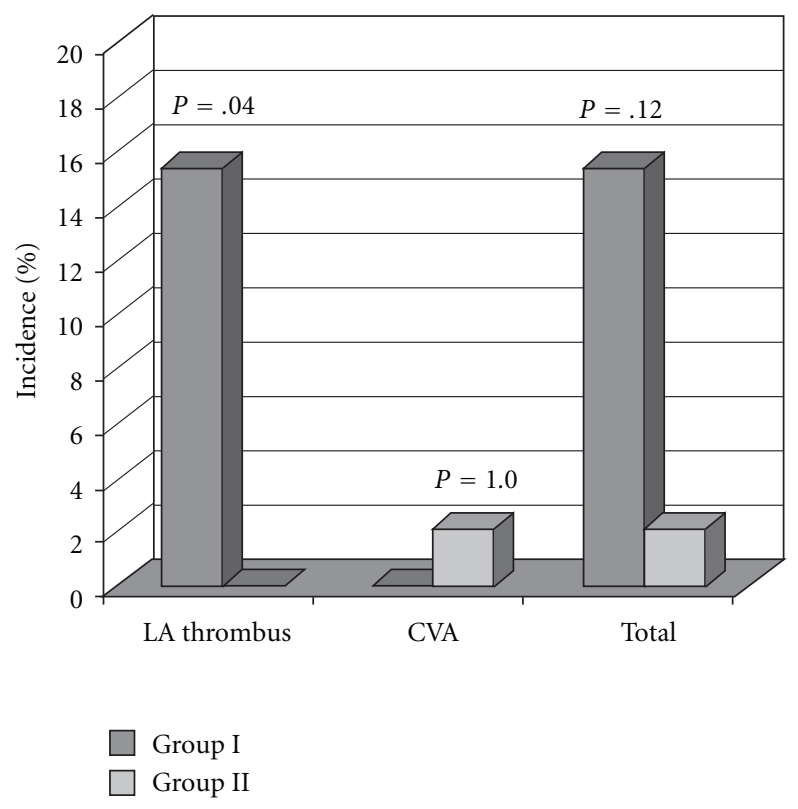

FIgUre 3: Histogram showing incidence of left atrial (LA) thrombi, cerebrovascular accidents (CVA), and the combined event rate. LA thrombi occurred more frequently in group I patients $(P=.04)$.

immediately after the procedure did not show signs of cerebral ischemia, repeat imaging on the next day revealed an ischemic area in the cerebellum. Fortunately, the patient fully recovered. In this patient, a thrombus in the left atrial appendage was seen 2.5 months before the procedure. He was then anticoagulated, and the repeat TEE before the procedure did not show an intracardial thrombus.

3.4. Total Event Rate. We observed intracardiac thrombi in 2 group I patients $(2 / 13 ; 15.4 \%)$ versus none in group II patients $(0 \% ; P=.04)$ and one CVA in one group II patient $(1 / 47 ; 2.1 \%)$ versus none in group I patient $(0 \%, P=\mathrm{ns})$, resulting in a total event rate of $15.4 \%$ in Group I versus $2.1 \%$ in group II $(P=.11)$ (Figure 3$)$.

\section{Discussion}

The use of effective anticoagulation is out of question during left atrial ablation procedures in order to minimize the risk of thrombus formation and embolism [5]. Despite the use of anticoagulation, the risk of periprocedural stroke reported ranges from $0 \%$ to $7 \%[5,9,10]$, with most of these occurring within $24 \mathrm{~h}$ of the ablation procedure. Predictors of periprocedural stroke seem to be a CHADS-Score $\geq 2$ and the history of a cerebrovascular accident [10]. Subclinical embolism as detected by magnetic resonance tomography in clinically asymptomatic patients has been reported in $11 \%$ of patients after left atrial ablation, with risk factors being left ventricular hypertrophy, left ventricular dilatation, and the presence of coronary artery disease [11].

It is common practice to start administration of heparin after transseptal access has been obtained safely, in order not to aggravate a possible pericardial effusion related to transseptal puncture. This approach is endorsed by a German consensus document on peri- and postinterventional stroke during cardiac catheter procedures [12]. However, the short dwell time of foreign material within the slow flow area of the left atrium may be sufficient for thrombus formation. Of note, measurements of ACTs in our study show that whenever the operator decides to administer heparin after transseptal access, the patient is exposed to an increased risk of left atrial thrombus due to suboptimal ACT levels (244 versus $301, P=.002$ ) for at least 15 minutes. The presented data show an incidence of $15.4 \%$ of thrombi adherent to sheaths and catheters within the left atrium almost immediately after left atrial access when heparin was given after transseptal puncture, as compared to an incidence of $0 \%$ when heparin was given before transseptal access. The most likely mechanism for thrombus formation is the presence of foreign material within the left atrium, since both thrombi (a) were adherent to sheaths and catheters, respectively, and (b) were detected early before energy delivery at a point when no endothelial disruption induced by ablation had occurred. Clinical characteristics previously described as risk factors for periprocedural stroke or subclinical embolism did not differ between these groups. Ren et al. found an incidence of left atrial thrombi as detected by ICE in $10.3 \%$ of patients, in all of whom heparin was given after transseptal access [13]. Our observed incidence is also comparable to another study, which found left atrial thrombi in $9.1 \%$ of cases, when heparin was given after the second transseptal puncture, as compared to $0 \%$ when heparin was given before transseptal access [14]. In this retrospective analysis, possible clinical risk factors of embolism as described above and the intensity of anticoagulation were not evaluated. However, our data and the data provided by Ren et al. [13] and Bruce et al. [14] strongly suggest that (a) ICE is useful in thrombus detection and (b) thrombus formation must be expected in $10-15 \%$ of cases when heparin is given after transseptal access.

In these studies, as well as in our population, removal of sheaths to the right atrium while applying suction and careful flushing was safe and did not provoke clinical evident embolism. Similarly to other authors, we believe that immediate withdrawal of the thrombus by retraction of sheaths and catheters, respectively, is the best strategy once a thrombus has been detected, since further manipulation of catheters carries the risk of thrombus dislodgement and embolism.

The only clinical evident embolism in our series of patients developed in a group II patient in whom no intracardiac thrombus was detected prior to embolism. The overall combined event rate of thrombus detection and clinical evident embolism therefore was not significantly different; however, this retrospective study might be underpowered to detect such a difference. Still, it might be that heparin administration, even if started early, might not be the optimal strategy for periprocedural anticoagulation. It remains unclear whether the observation of an intracardiac thrombus is predictive of embolism, however, it seems to be intuitive to avoid such a thrombus formation. An association of left atrial thrombus and embolism was also not be found in other studies $[13,14]$, which may be related to (a) the 
small number of LA thrombi overall detected, (b) the early detection by ICE and subsequent removal, or (c) subclinical embolism. Of note, subclinical embolism has been described with an equivalent incidence as LA thrombi [11].

Continued oral anticoagulation with a therapeutic INR at the time of ablation is another strategy for peri-interventional management of anticoagulation. Low event rates of embolism as well as bleeding have been described in experienced centers, and this evidence supports the strategy of maintaining therapeutic levels of oral anticoagulation during ablation procedures [15-17]. Current evidence also shows that cardiac tamponade is not more frequent, more severe, or more difficult to manage when occurring during continued oral anticoagulation $[17,18]$.

\section{Conclusions}

Intracardiac thrombus formation is frequent when heparin is administered after transseptal access and not associated with previously described risk factors for clinical and subclinical embolism. Early administration of heparin (i.e., before transseptal access) diminishes this risk but did not affect the rate of CVAs in this study. Further prospective investigation should address the issue whether early peri-interventional anticoagulation reduces the risk of embolism and stroke.

5.1. Limitations. The study results must be appraised according to the retrospective and nonrandomized study design and the low event rate. Additionally, despite consequent observation of the ICE images, as a two-dimensional imaging modality, ICE cannot continuously monitor the whole body of the left atrium. Therefore, we cannot exclude that thrombi developed in an unmonitored part of the left atrium and thus would have been overlooked.

\section{References}

[1] M. Haïssaguerre, P. Jaïs, D. Shah et al., "Spontaneous initiation of atrial fibrillation by ectopic beats originating in the pulmonary veins," New England Journal of Medicine, vol. 339, no. 10, pp. 659-666, 1998.

[2] H. Calkins, M. R. Reynolds, P. Spector et al., "Treatment of atrial fibrillation with antiarrhythmic drugs or radiofrequency ablation: two systematic literature reviews and meta-analyses," Circulation Arrhythmia and Electrophysiology, vol. 2, no. 4, pp. 349-361, 2009.

[3] E. R. Ellis, S. D. Culler, A. W. Simon, and M. R. Reynolds, "Trends in utilization and complications of catheter ablation for atrial fibrillation in Medicare beneficiaries," Heart Rhythm, vol. 6, no. 9, pp. 1267-1273, 2009.

[4] J. D. Fisher, M. A. Spinelli, D. Mookherjee, A. K. Krumerman, and E. C. Palma, "Atrial fibrillation ablation: reaching the mainstream," Pacing and Clinical Electrophysiology, vol. 29, no. 5, pp. 523-537, 2006.

[5] H. Calkins, J. Brugada, D. L. Packer et al., "HRS/EHRA/ECAS expert consensus statement on catheter and surgical ablation of atrial fibrillation," Europace, vol. 9, no. 6, pp. 335-379, 2007.

[6] W. Saliba and J. Thomas, "Intracardiac echocardiography during catheter ablation of atrial fibrillation," Europace, vol. 10, 3, pp. iii42-iii47, 2008.
[7] S. Asbach, J. Biermann, P. Blanke, G. Pache, C. Bode, and T. S. Faber, "Incidental finding of a pulmonary embolus by intracardiac echocardiography during an atrial fibrillation ablation procedure," Europace, vol. 12, pp. 1199-1200, 2010.

[8] J. M. Kalman, J. E. Olgin, M. R. Karch, and M. D. Lesh, "Use of intracardiac echocardiography in interventional electrophysiology," Pacing and Clinical Electrophysiology, vol. 20, no. 9 I, pp. 2248-2262, 1997.

[9] D. Patel, S. M. Bailey, A. J. Furlan et al., "Long-term functional and neurocognitive recovery in patients who had an acute cerebrovascular event secondary to catheter ablation for atrial fibrillation," Journal of Cardiovascular Electrophysiology, vol. 21, no. 4, pp. 412-417, 2010.

[10] D. Scherr, K. Sharma, D. Dalal et al., "Incidence and predictors of periprocedural cerebrovascular accident in patients undergoing catheter ablation of atrial fibrillation," Journal of Cardiovascular Electrophysiology, vol. 20, no. 12, pp. 13571363, 2009.

[11] J. W. Schrickel, L. Lickfett, T. Lewalter et al., "Incidence and predictors of silent cerebral embolism during pulmonary vein catheter ablation for atrial fibrillation," Europace, vol. 12, no. 1, pp. 52-57, 2010.

[12] J. Röther, U. Laufs, M. Böhm et al., "Consensus paper on periinterventional and postinterventional stroke during cardiac catheter procedures," Nervenarzt, vol. 80, no. 10, pp. 1205$1215,2009$.

[13] J. F. Ren, F. E. Marchlinski, and D. J. Callans, "Left atrial thrombus associated with ablation for atrial fibrillation: identification with intracardiac echocardiography," Journal of the American College of Cardiology, vol. 43, no. 10, pp. 1861-1867, 2004.

[14] C. J. Bruce, P. A. Friedman, O. Narayan et al., "Early heparinization decreases the incidence of left atrial thrombi detected by intracardiac echocardiography during radiofrequency ablation for atrial fibrillation," Journal of Interventional Cardiac Electrophysiology, vol. 22, no. 3, pp. 211-219, 2008.

[15] A. A. Hussein, D. O. Martin, W. Saliba et al., "Radiofrequency ablation of atrial fibrillation under therapeutic international normalized ratio: a safe and efficacious periprocedural anticoagulation strategy," Heart Rhythm, vol. 6, no. 10, pp. 14251429, 2009.

[16] O. M. Wazni, S. Beheiry, T. Fahmy et al., "Atrial fibrillation ablation in patients with therapeutic international normalized ratio: comparison of strategies of anticoagulation management in the periprocedural period," Circulation, vol. 116, no. 22, pp. 2531-2534, 2007.

[17] L. Di Biase, J. D. Burkhardt, P. Mohanty et al., "Periprocedural stroke and management of major bleeding complications in patients undergoing catheter ablation of atrial fibrillation: the impact of periprocedural therapeutic international normalized ratio," Circulation, vol. 121, no. 23, pp. 2550-2556, 2010.

[18] R. Latchamsetty, S. Gautam, D. Bhakta et al., "Management and outcomes of cardiac tamponade during atrial fibrillation ablation in the presence of therapeutic anticoagulation with warfarin," Heart Rhythm, vol. 8, no. 6, pp. 805-808, 2011. 


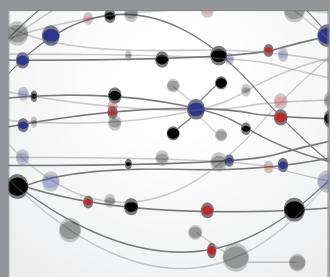

The Scientific World Journal
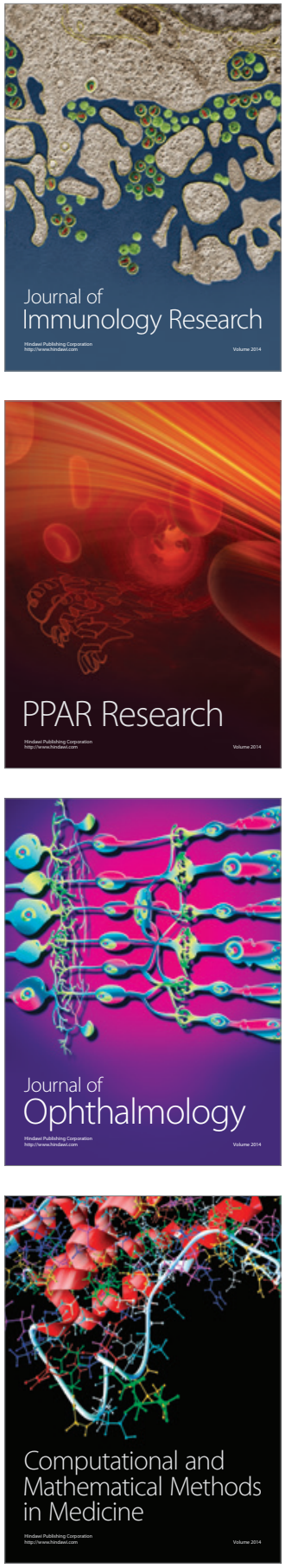

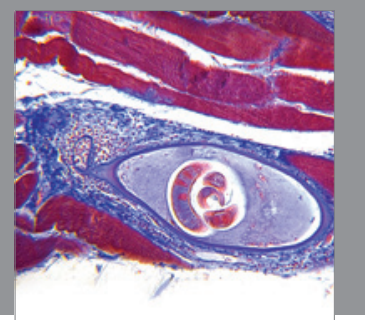

Gastroenterology

Research and Practice
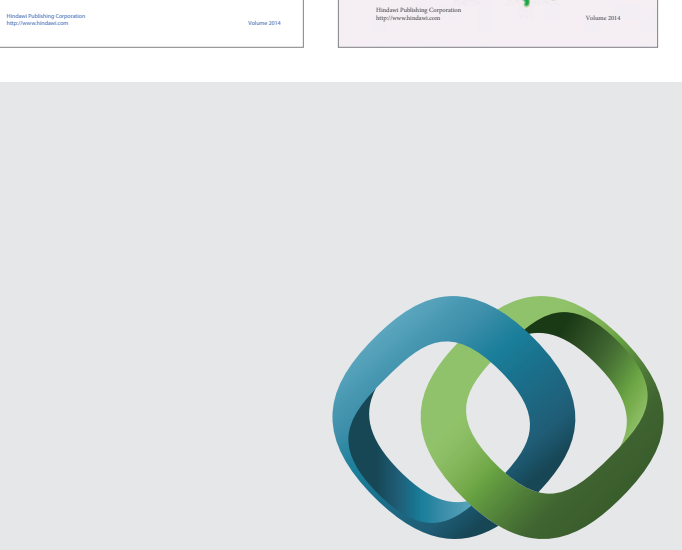

\section{Hindawi}

Submit your manuscripts at

http://www.hindawi.com
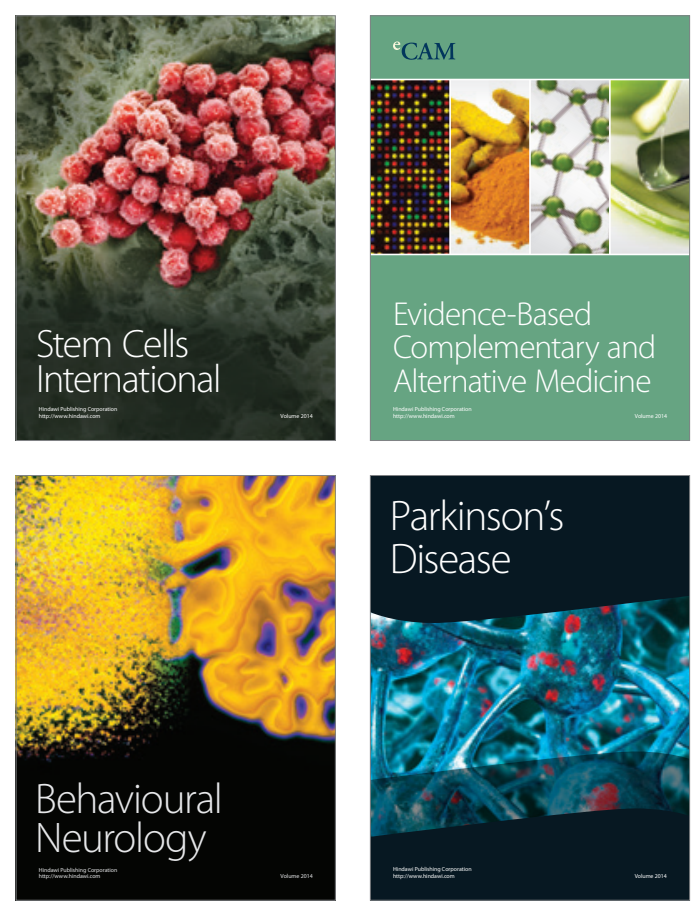

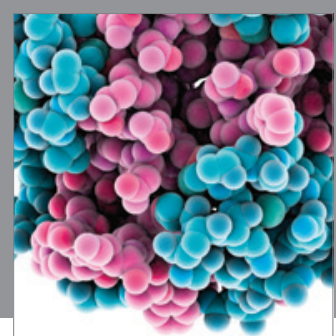

Journal of
Diabetes Research

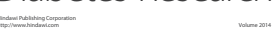

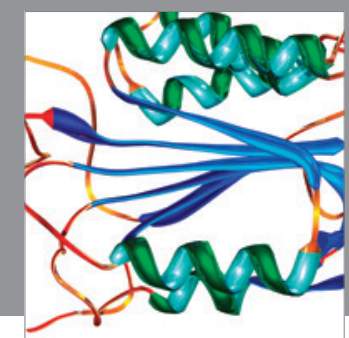

Disease Markers
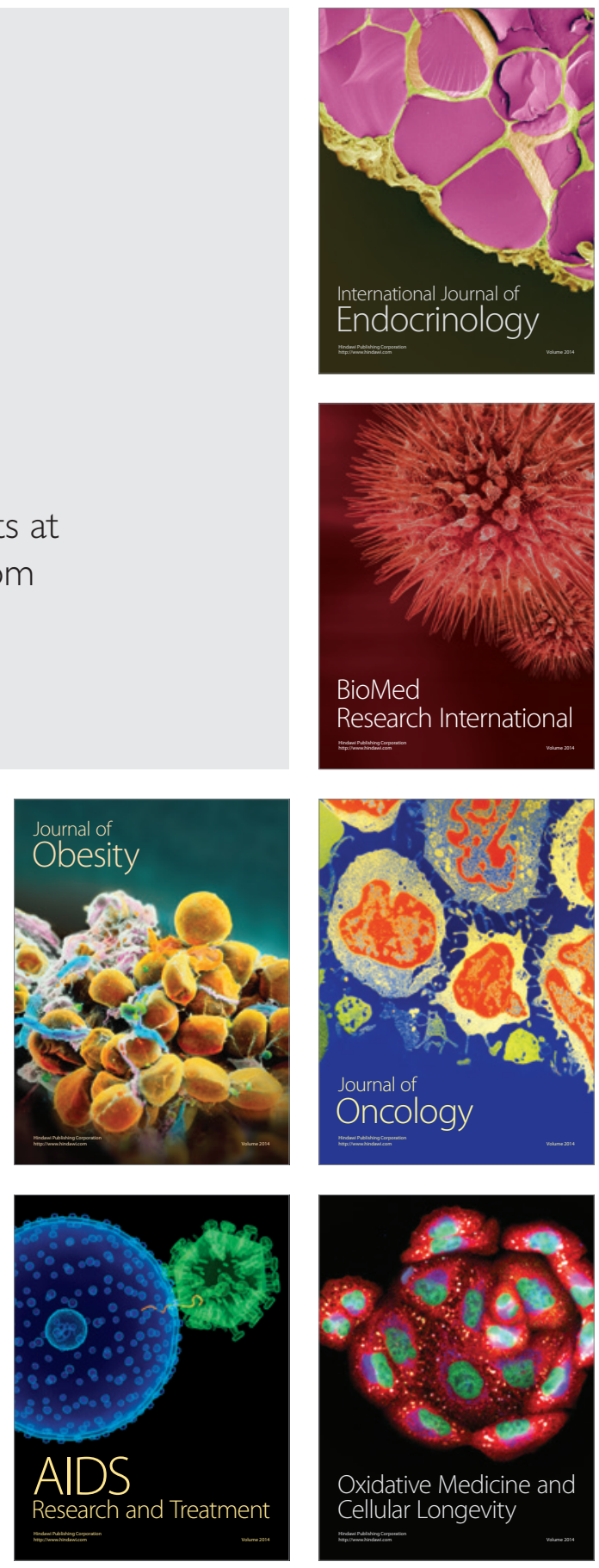